\title{
Microbiomes: A Possible Space Component?
}

\author{
Chandra Wickramasinghe ${ }^{1,2,3 *}$, Ananda Nimalasuriya ${ }^{4}$, Milton Wainwright ${ }^{5}$ and Gensuke Tokoro ${ }^{2}$ \\ ${ }^{1}$ Buckingham Centre for Astrobiology, University of Buckingham, Buckingham, UK \\ ${ }^{2}$ Institute for the Study of Panspermia and Astro-economics, Gifu, Japan \\ ${ }^{3}$ University of Peradeniya, Peradeniya, Sri Lanka \\ ${ }^{4}$ Kaiser Permanente Riverside Medical, 10800 Magnolia Ave \# 1, Riverside, CA 92505, USA \\ ${ }^{5}$ Department of Molecular Biology and Biotechnology, University of Sheffield, Sheffield, UK
}

"Corresponding author: Chandra Wickramasinghe, Buckingham Centre for Astrobiology, University of Buckingham, Buckingham, UK, Tel: +44-777-838-9243; E-mail: ncwick@gmail.com

Rec date: September 17, 2015; Acc date: September 24, 2015; Pub date: September 28, 2015

Copyright: (c) 2015 Chandra Wickramasinghe, et al. This is an open-access article distributed under the terms of the Creative Commons Attribution License, which permits unrestricted use, distribution, and reproduction in any medium, provided the original author and source are credited.

\begin{abstract}
We hypothesize that components of microbiomes associated with humans and higher life-forms may be continually replenished from space. Examination of stratospheric dust using culture-independent gene-mapping techniques could reveal their possible existence and thus demonstrate unequivocally our evolutionary connection with the external universe.
\end{abstract}

Keywords: Microbiome; Evolution; Panspermia; Comets; Stratospheric dust

\section{Introduction}

"Once you eliminate the impossible, whatever remains, no matter how improbable, must be the truth." - Sir Arthur Conan Doyle

The role of symbiotic microorganisms, particularly in the human gut has been recognized for many years. The overwhelming importance of the microbiome, corresponding to an estimated total bacterial count of some $\sim 10^{14}$ (100 trillion) individual cells has, however, been relatively recent [1]. Advances in culture-independent gene sequencing techniques have made possible a better understanding of this somewhat enigmatic entity although its origin and evolutionary impact remains unclear $[2,3]$.

Automated gene sequencing techniques have yielded a total number of genes associated with our microbiome that grossly exceeds the 22,000 or so protein coding genes in the human genome. A consortium of researchers has recently published a gene catalogue of $3.3 \times 10^{6}$ nonredundant genes in the microbiome of the human gut $[3,4]$. Since gene sequence mapping does not distinguish between viruses, plasmids and transposable genes, the viral component in the microbiome may perhaps be the most significant.

The human gut microbiota has often been described as the most densely populated ecosystem on Earth $[5,6]$. Gut bacteria divide into two broad classes: commensual or symbiotic organisms, and dysbiotic or pathogenic organisms. Included within these classes are many groups of extremophiles, including acidophiles, that are significantly different from free-living microbial populations. Most importantly they have the proven ability to transfer viral particles (virions) to invading bacteria. The remarkable individuality and time variability of the human microbiome, particularly in the gut [7], has also come as a surprise to investigators in recent years. Its possible role in health, immunity and disease is only just coming to be recognized by physicians. Some human diseases that have defied understanding may well be connected with properties of the microbiome. For instance it has been suggested that patients with schizophrenia have a significantly altered microbiome in their mouths and throats [8].

How did the human (and other) microbiomes arise in the first place? Is it the outcome of millions of years of co-evolution between evolved organisms (mammals, humans) and an ever-changing population of environmental bacteria and viruses? Or is a population of microbial/viral entities identifiable with microbiomes continuously being replenished from space?

A discovery possibly related to microbiomes is the realization that the total biomass of our planet may be dominated by viruses. Epifluorescence microscopy of sea-water has recently turned up with a total count of $0.5-1 \times 10^{10}$ virions per litre or $\sim 10^{31}$ virions throughout the oceans. The possibility that microbiomes associated with animals are acquired, at least in part, from this pool of viruses cannot be ignored. Nor can we dismiss the even more contentious possibility that components of microbiomes form part of a continuing ingress of biomaterial from comets. From 2001 onwards evidence for an ongoing entry of biological entities from comets, consistent with Panspermia [9] has grown to the point of being close to compelling $[10,11]$. The case for Panspermia has been further strengthened by recent discoveries of exoplanets and the diminishing average distance between neighboring habitable planets [12].

Stratospheric samples of presumed cometary origin recovered from $41 \mathrm{~km}$ in the stratosphere have shown evidence for both culturable and non-culturable microorganisms. The non-culturable component has been detected in great abundance by fluorescence techniques that show positive for both the presence of DNA/RNA and membrane potential (Figure 1). It is possible that clumps such as these are indeed entire microbiomes or parts thereof that would eventually become incorporated in terrestrial life forms.

Although a few culturable microorganisms have been isolated and their 16s-RNA sequenced $[13,14]$ there remains an ambiguity as to 
whether such single bacteria of sizes less than 1 micrometre may somehow have been lofted from the ground.

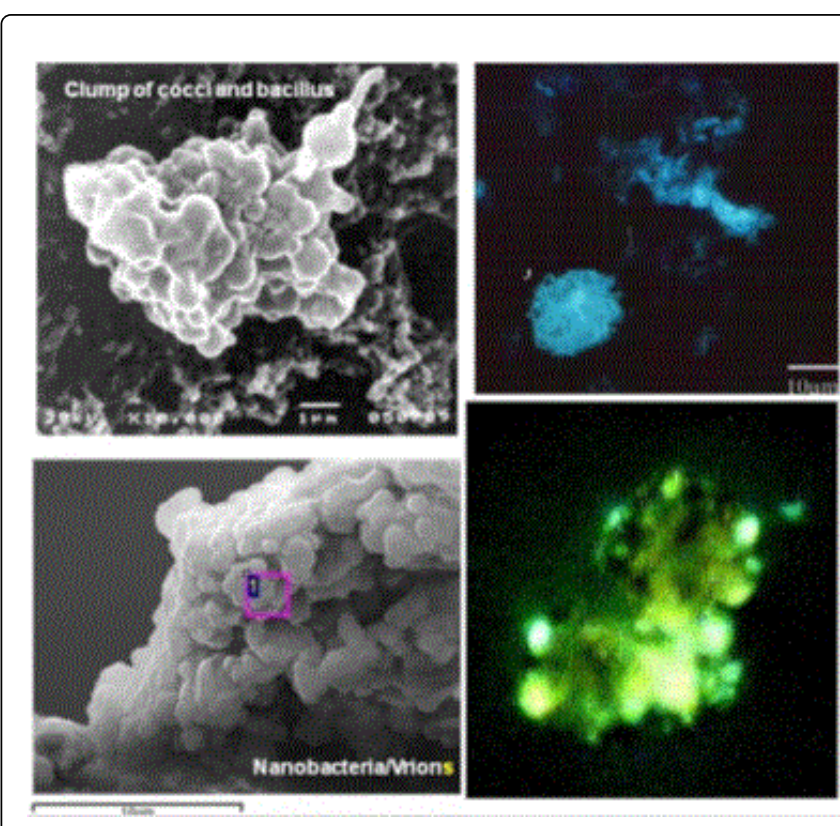

Figure 1: Left panels: Carbon/oxygen rich biological structures of diameters greater than 10 micometres.

Right panels: top-amorphous 10-20 micrometre clumps of biological particles detected by DNA staining techniques; bottom large clump of bacterial cells detected by stain (carbocyanine) revealing membrane potential. [10].

On the other hand the much larger clumps of biomaterial (bacteria/ nanobacteria/viruses) detected both by electron microscopy and staining techniques (Figure 1) are decisively far too heavy to be lofted from the ground. So we can safely assume that they fall from space. Such clumps could include bacteria as well as RNA/DNA viruses that could interact with and augment microbiomes associated with terrestrial creatures including humans.

In the balloon experiments that are being planned at the Institute for the Study of Panspermia and Astroecomics infalling cometary material will be collected at regular intervals. Samples of the collected material will be used for various tests, and the hope is to include techniques that would seek evidence of microbiome-related genome components in stratospheric dust.

Based on our 2001 sampling of the stratosphere at $41 \mathrm{~km} \mathrm{[10]} \mathrm{we}$ estimate a daily input of biomaterial over the entire Earth of $\sim 0.3$ tonne. With a typical virion mass of $10^{-21} \mathrm{~g}$ we would thus have $\sim 3 \times$ $10^{26}$ virions per day. On this basis the total virion count in the oceans of $\sim 10^{31}$ virions would be replaced on a timescale of $3 \times 10^{4}$ days $\sim 100$ $\mathrm{yr}$, or 3 human generations. Over such a timescale microbiome-related virions if they are incident from space can act as horizontal gene transfer (HGT) agents and play a crucial role in host evolution [9]. One might speculate that the evolution of hominids over millions of years was driven by the accumulation of such virions into their genomes $[9,15,16]$.

On a much shorter time interval the interactions between microbiome-related virions and host species might be used to practical advantage in the field of medicine. For example it is known that gene transfer from the microbiome to an invading pathogen would sometimes vitiate the effect of antibiotics and vaccines and permit its ingress to the host [17]. We could speculate that it may be possible for strategies for vaccines as well as antibiotics to be developed in response to virions collected in the stratosphere.

For a sample of biomaterial recovered from the stratosphere (e.g. clusters in Figure 1) techniques of metagenomics, now routinely available on a commercial basis, offer the possibility of rapidly "deconstructing" collective genomes of bacteria and viruses. The discovery of novel sequences that may be occasionally present could give a clue as to their space origin, thus establishing consistency with ongoing Panspermia.

In conclusion we emphasize that we present this proposal as a potentially falsifiable proposition. It may at first sight appear improbable, but the technology to disprove it exists, and in our view it is our duty as scientists to deploy it to this end.

\section{References}

1. Turnbaugh PJ, Ley RE, Hamady M, Liggett CF, Knight R, et al. (2007) The human microbiome project. Nature 449: 804-810.

2. Peterson J, Garges S, Giovanni M, McInnes P, Wang L, et al. (2009) The NIH Human Microbiome Project. Genome Research 19: 2317-2323.

3. Qin J, Li R, Raes J, Arumugam M, Burgdorf KS, et al. (2010) A human gut microbial gene catalogue established by metagenomic sequencing. Nature 464: 59-65.

4. Consortium IHGS. (2004) Finishing the euchromatic sequence of the human genome. Nature 431: 931-945.

5. Ley RE, Lozupone CA, Hamady M (2008) Worlds within worlds: evolution of the vertebrate gut microbiota. Nature Reviews Microbiology 6: 776-788.

6. Penders J, Stobberingh EE, Savelkoul PH, Wolffs PF (2013) The human microbiome as a reservoir of antimicrobial resistance. Microbiol 4: 87.

7. Turnbaugh PJ, Hamady M, Yatsunenko T, Brandi LC, Alexis D, et al. (2009) A core gut microbiome in obese and lean twins. Nature 457: 480484.

8. Dinan TG, Borre YE, Cryan JF (2014) Genomics of schizophrenia: time to consider the gut microbiome? Molecular Psychiatry 19: 1252-1257.

9. Hoyle F, Wickramasinghe NC (1981) Comets and the Origin of Life. World Scientific Publishing Co Pvt Ltd.

10. Harris MJ, Wickramasinghe NC, Lloyd D, Narlikar JV, Rajaratnam P, et al. (2002) Detection of living cells in stratospheric samples. Proc. SPIE 4495: 192.

11. Wainwright $\mathrm{M}$, Rose CE, Baker AJ, Wickramasinghe NC, Omairi $\mathrm{T}$ (2015) Biological Entities Isolated from Two Stratosphere LaunchesContinued Evidence for a Space Origin. J Astrobiol Outreach 3: 2 .

12. Wickramasinghe C, Smith W (2014) Convergence to Panspermia. Hypothesis Journal 12: 1 .

13. Shivaji S, Chaturvedi P, Begum Z, , et al. (2009) Janibacter hoylei sp. nov., Bacillus isronensis sp. nov. and Bacillus aryabhattai. sp. nov., isolated from cryotubes used for collecting air from the upper atmosphere. Int J Syst Evol Microbiol 59: 2977-2986

14. Pawar SP, Dhotre DP, Shetty SA, Chowdhury SP, Chaudhari BL, et al. (2012) Genome sequence of Janibacter hoylei MTCC8307, isolated from the stratospheric air. J Bacteriol 194: 23.

15. Ryan F (2009) Virolution. Harper Collins Publishers, London.

16. Wickramasinghe NC (2013) DNA sequencing and predictions of the cosmic theory of life, Astrophys Sp Sci 343: 1-5

17. Sommer MO, Church GM, Dantas G (2010) The human microbiome harbors a diverse reservoir of antibiotic resistance genes. Virulence 1: 299-303. 